\title{
NEURAL NETWORKS AS A TOOL FOR GEORADAR DATA PROCESSING
}

\author{
PiOTR SZYMCZYK ${ }^{a, *}$, SYLWIA TOMECKA-SUCHOŃ ${ }^{b}$, MAGDALENA SZYMCZYK $^{a}$ \\ ${ }^{a}$ Department of Automatics and Biomedical Engineering \\ AGH University of Science and Technology, al. A. Mickiewicza 30, 30-059 Kraków, Poland \\ e-mail: \{piotr.szymczyk, magdalena.szymczyk\} @agh.edu.pl] \\ ${ }^{b}$ Department of Geophysics \\ AGH University of Science and Technology, al. A. Mickiewicza 30, 30-059 Kraków, Poland \\ e-mail: tomecka@agh.edu.pl
}

\begin{abstract}
In this article a new neural network based method for automatic classification of ground penetrating radar (GPR) traces is proposed. The presented approach is based on a new representation of GPR signals by polynomials approximation. The coefficients of the polynomial (the feature vector) are neural network inputs for automatic classification of a special kind of geologic structure-a sinkhole. The analysis and results show that the classifier can effectively distinguish sinkholes from other geologic structures.
\end{abstract}

Keywords: neural networks, artificial neural networks, ground penetrating radar, classification of a geological structure, sinkhole.

\section{Introduction}

Among various risks, relatively little attention has been paid to the consequences of voids migration towards the surface of fracture zones in a rock mass. This issue, however, is very important since the effects of sinkhole migration take place rapidly, constituting a potential danger for human beings, animals and construction objects. The presence of such zones in the rock mass is a result of anthropogenic activities, mining in particular. Such phenomena have been observed in the mining areas in Poland. A considerable part of the Western Małopolska region is occupied by active coal mines, part of which have been closed, with discontinuities migrating in the rock mass as a consequence (Marcak et al., 2008).

The paper is based on the results of field studies in the post-mining sinkhole area in Trzebinia-Siersza. Siersza is known for hard coal extraction. The coal mine Siersza, operating since the end of the 18th century, was closed in 2001, but the consequences of its activity have been observed in the form of constantly appearing sinkholes.

The development of non-elastic strains in the neighborhood of post-mining voids leads to higher water content in the top part of the void and a loose layer zone.

*Corresponding author
The water flowing through the porous medium destroys it. At the disintegration moment, the most severely destroyed fragments fall down onto the bottom of the void. Thus, the described mechanism explains the slow movement of the void towards the surface.

The fracture zone has higher porosity and water saturation; therefore, the dielectric properties of the rock change and georadar anomalies are generated. Georadar surveys allow distinguishing between stable anomalous regions and those which develop into the sinkholes.

In the absence of systems for automated interpretation of georadar data, so far they have been interpreted only on the basis of data visualization. Image processing has also been used to process GPR scatters but they are not effective for this type of data. The proposed system of automated interpretation and description of the results will allow the crew to survey much larger areas, shorten the time of the procedure, lower the costs and significantly increase the accuracy.

\section{Subject matter}

The paper presents a feasibility analysis of neural networks as a tool for the analysis, processing and interpretation of georadar signals. 


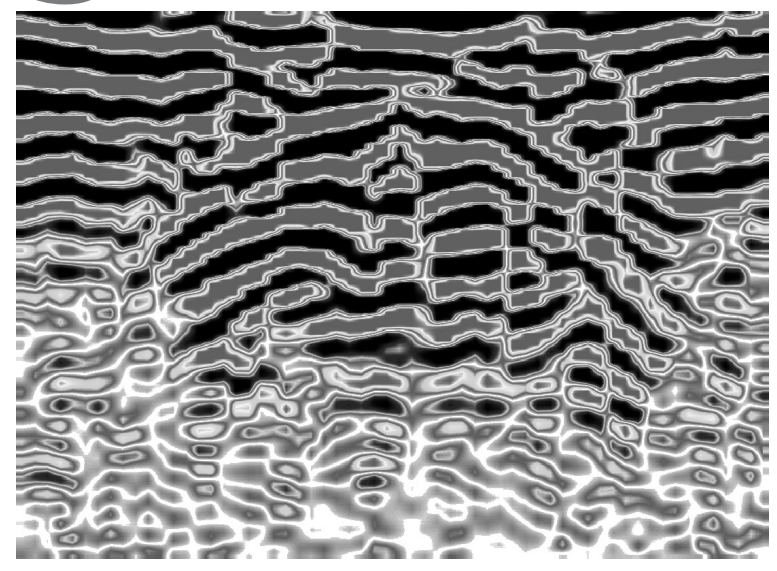

Fig. 1. Sample georadar image.

First, let us explain why to choose neural networks for this purpose. Most importantly, neural networks are very efficient learning tools. Georadar signals are known to bring information about geologic structures underground on the basis of propagation and reflections (interface of layers of different relative permittivity) of signals in the form of electromagnetic waves. As a result, we are given spectacular images of georadar signals, e.g., Fig. 11 but their interpretation is not simple.

This stems from the complex character of physical phenomena taking place when electromagnetic waves are propagating and reflecting in the ground, and also from the fact that particular ground penetrating radar (GPR) devices provide images of diversified properties (McClymont et al., 2008; Miaskowski and Cieszczyk, 2011; Wei-Li et al., 2012). As a result, even great specialists, well trained in evaluating georadar signals obtained with one type of GPR device, have to learn how to interpret correctly results delivered by another GPR device. Analogously, computer tools responsible for the acceleration, automation and objectivization of georadar signals interpretation have to learn, too. Presently, neural networks are selected for enhancing the process of georadar data interpretation.

\section{Use of neural networks for georadar profiles interpretation}

Neural networks are a tool of a specific mode of operation (Tadeusiewicz et al., 2014), as visualized in Fig. 2 Prior to using neural networks for georadar signals interpretation, taking advantage of all their possibilities and properties, one should define all the elements generally presented in Fig. 2 The first issue to be solved is the problem of representing georadar data on the neural network input. An image, e.g., the one presented in Fig. 1 cannot be directly put at the neural network input. Apart from other, more subtle, reasons, we must

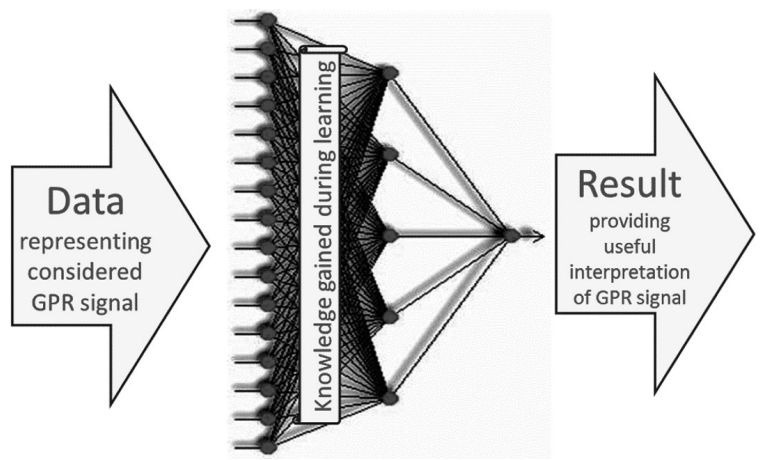

Fig. 2. General concept of using neural networks for solving the analyzed task.

remember that the image consists of an immense number (hundreds of thousands) of voxels; therefore, the input layer of a network which would accept such an image should have the same number of neurons, and this would create problems. Even bigger issues would arise while relating such a large input layer with the correspondingly rich hidden layer (a layer of neurons analyzing the data). This would require establishing values of at least a few millions of weight coefficients at the learning stage of the network. Technically this cannot be performed.

This issue is known from other applications of neural networks, which are connected with computer processing, analysis and pattern recognition (Tadeusiewicz, 2011; 2010). There are occasionally used methods of dividing images into windows or regions of interest (ROIs). These methods are sometimes used for ordinary images, though they are completely impracticable for georadar images. This is mainly due to the contextual relations between voxels in the GPR, which are completely different from relations between pixels used, e.g., for coding in JPG, characteristic of regular visual images.

While looking for a method of a correct georadar image representation in a neural network, attention has been paid to the fact that a GPR image (profile) consists of a set of traces, as shown in Fig. 3, where the typical echogram (radargram) is presented in the Wiggle format. In this format, the results of particular surveys are shown as lines running from the top to the bottom. Their diffractions from the ideal vertical direction correspond to the successive echoes reached by the GPR device, being a consequence of reflections of electromagnetic waves from successive geologic structures. Diffractions to one side represent waves reflecting in line with the emitted signal, whereas the diffractions oriented in the opposite direction correspond to counterphase reflections. The amplitude of diffractions informs about the energy of the reflected wave.

Each trace corresponds to the result of one georadar survey performed in the successive point of the trajectory 


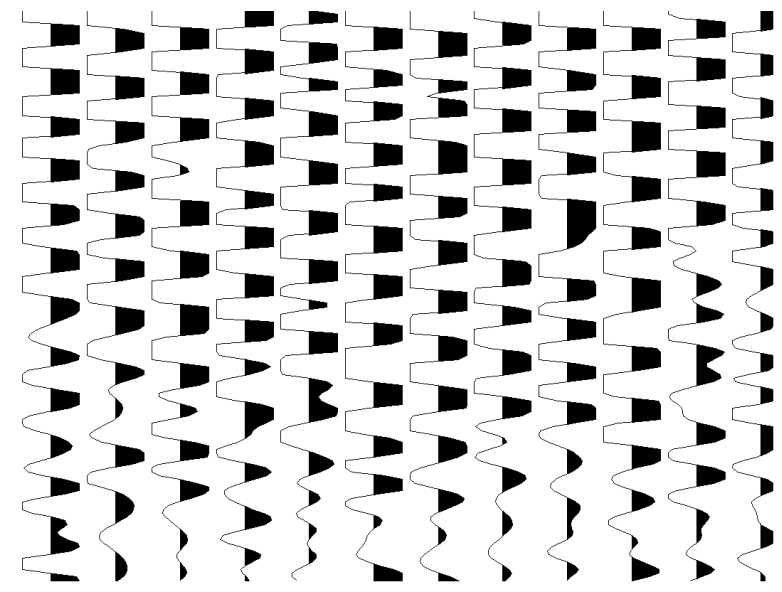

Fig. 3. Georadar image as a collection of traces.

along which the GPR device was drawn. The locations of particular measurement points are presented on the horizontal axis as a variable $x$ standing for a distance between the starting point (place of the first survey). The vertical size of the trace represents differentiated reflections of electromagnetic waves obtained from various depths. This is connected with the fact that reflections mainly come from the boundaries of geologic structures localized at different depths. This is symbolized by the DEPTH variable on the vertical axis on the right-hand side of the figure. It is worth noticing that the values of this variable are approximate; only delays of electromagnetic echo are determined accurately by GPR in relation to the moment of emitting the scanning signal (the variable $t$ on the vertical axis on the left side). The values of a hypothetical $D E P T H$, at which the reflection took place, are calculated for the assumed (identical for the entire way of the signal) propagation velocity of the electromagnetic wave in the ground. This velocity in a definite case is generally unknown and, what is even more, it changes in reality because particular geologic structures encountered by the electromagnetic waves have different dielectric constants; i.e., the propagation velocity of electromagnetic waves is different. Fortunately, a detailed depth of the analyzed and evaluated geologic structure does not matter in the analyzed case.

A profile consisting of 645 traces was used in these investigations. In the digital representation each trace contained 416 measurement points (a total of 268320 voxels). The 416-point vector was too big to be introduced to the network input; therefore, a signal representation was searched for to represent its most important features and significantly reduce the size of the input vector. This effect could be achieved by determining the aim of the interpretation of analyzed georadar signals more accurately. In the analyzed case the task was to find out the underground void, i.e., the result of an incorrectly finished mining activity. The presence of such a void underground and its systematic migration towards the surface creates hazard for construction objects, e.g., buildings or roads, and also for people.

The traces obtained by the georadar had to be classified as informing about voids or neutral. Therefore, various input signals entering the neural network had to contain information about the general shape of the signal coming from the analyzed trace, as this shape was assumed to carry information about the void. However, they should not depend on, e.g., the way in which the characteristic features of the shape are located with respect to the vertical axis (the void should be detectable regardless of the depth at which it occurs).

An original (and unprecedented in the specialist literature) way of representing signals at the neural network input was proposed, consisting in approximating the profile of the signal with a polynomial. Then, attempts were made to use the coefficients of the polynomial as a signal entered at the neural network input. The procedure can be formally described on the basis of the following model.

Let us denote the profile of a GPR signal along the analyzed trace at a point of a coordinate $x$ as $G_{x}(d)$, where $d$ is the depth from which the echogram signal is received. Let us assume that signal $G_{x}(d)$ can be approximated by an $N$-th degree polynomial with coefficients $a_{0 x}, a_{1 x}, a_{2 x}, \ldots, a_{N x}$. This can be symbolically written as

$$
G_{x}(d) \cong \sum_{i=0}^{N} a_{i x} d^{i} .
$$

Following the approximation, one may assume that a signal $G_{x}(d)$ at the neural network input is represented by a vector $a$ with $N+1$ elements $a_{0 x}, a_{1 x}, a_{2 x}, \ldots, a_{N x}$.

It was empirically confirmed that the optimum degree of an approximation polynomial is $N=20$. At lower values of the degree, the approximation was inaccurate and certain subtle features of the trace were insufficiently well represented at the neural network input. When selecting the degree $N>20$, a considerable part of the coefficients $a_{i x}$ for high values of $i$ equalled zero, proving that a polynomial of such a high degree was not necessary for this type of approximation.

The coefficients of a polynomial determined in this way can be exemplified as $a_{0 x}, a_{1 x}, a_{2 x}, \ldots, a_{N x}$ for trace no. 27 in the area where no underground void occurs, and for trace no. 330 in the area where the presence of a void was confirmed by a sinkhole formed months later. The comparison of particular coefficients shows how well the presented signal representation stands for features important to our investigation.

After establishing how the input of a network generally presented in Fig. 2 is going to be represented, 
Table 1. Polynomial coefficients for two selected traces.

\begin{tabular}{|c|c|c|}
\hline Trace no. & 27 & 330 \\
\hline \hline$a_{0}$ & -0.3505 & 0.2699 \\
$a_{1}$ & 0.3331 & -0.0028 \\
$a_{2}$ & 4.8442 & -4.0662 \\
$a_{3}$ & -4.3115 & 0.2897 \\
$a_{4}$ & -28.4144 & 25.8351 \\
$a_{5}$ & 23.4807 & -3.0743 \\
$a_{6}$ & 92.0963 & -90.1236 \\
$a_{7}$ & -69.9186 & 13.8706 \\
$a_{8}$ & -179.8618 & 188.2779 \\
$a_{9}$ & 123.7323 & -33.1554 \\
$a_{10}$ & 216.4069 & -241.0737 \\
$a_{11}$ & -132.3002 & 44.7143 \\
$a_{12}$ & -157.3952 & 185.9049 \\
$a_{13}$ & 83.0048 & -33.7151 \\
$a_{14}$ & 65.0304 & -81.3861 \\
$a_{15}$ & -28.1220 & 13.2299 \\
$a_{16}$ & -13.4095 & 17.8733 \\
$a_{17}$ & 4.2997 & -2.2748 \\
$a_{18}$ & 1.0421 & -1.5068 \\
$a_{19}$ & -0.1884 & 0.1113 \\
$a_{20}$ & 0.0717 & 0.0902 \\
\hline
\end{tabular}

the desired network output was considered. It was assumed that a binary output (void or no void) will not be useful in our task as too categoric solutions will be obtained at a considerably high level of error. Therefore, instead of a classification variant, a regression network was assumed, and the input signal from the network was to determine the probability that the analyzed trace corresponds to a void.

\section{Selection of the neural network structure and its learning}

In all handbooks on neural network application, attention is paid to the proper selection of the network structure. In the analyzed task we had to establish the type of network, the number of layers, and the number of neurons in particular layers. This aim was realized with the use of a specialized computer tool, i.e., the module of automatic network designer, being part of the package Statistica Neural Networks by StatSoft. The structure of the network was proposed after analyzing available data and testing several dozen of potentially possible structures. It consisted of 21 neurons in the input layer (the degree of the polynomial equalled 20 as input data characterizing the analyzed georadar signal trace), and one hidden layer with 10 neurons of sigmoid functions and with a single (also sigmoid) neuron in the output layer. This type of network was described in the literature as a multi-layer perceptron (MLP). Its structure is presented in Fig. 4 with the use of symbols as in the package Neural Network Toolbox of the Matlab environment (this tool was employed in the process of network learning and for investigating its usability, especially the Neural Network Pattern Recognition Tool).

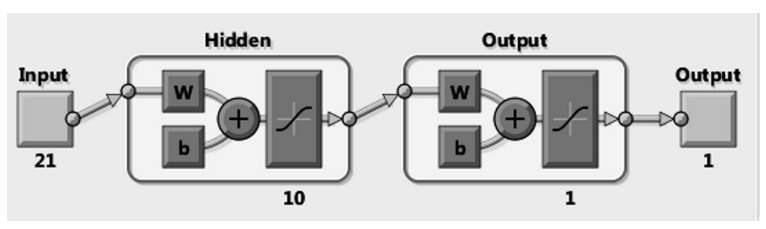

Fig. 4. Structure of the neural network applied.

The described network underwent the process of learning. For this reason, a set of georadar data were collected. They came from probings in an area of a potential underground void, which was later confirmed by a large sinkhole. Relating places of previous georadar probings with certainty about the manifested void, each trace in the analyzed profile was ascribed an attribute saying whether the trace should reveal a void (which actually was there!) or a 'regular' geologic structure. Thus, the prepared data covered a total of 645 traces, which were later used for training the networks.

These data, in congruence with the principles of the neural network, were split into three parts: $70 \%$ of possessed data (451 traces) were used directly for network learning (learning set), 15\% (97 traces) for validation and the protecting against the overlearning effect (validation set), and the remaining 15\% (97 traces) for tests checking out the generalization capacity of the network (test set).

Learning followed the scaled conjugate gradient algorithm. The conjugate gradient method was chosen because it is the most prominent and effective iterative method for solving systems of linear equations. The maximum learning time was assumed to be lower than 1000 epochs. In reality, the time was shortened by the anti-overlearning condition, checked out after each learning epoch with a validation set. The image of the network while it learns in the Matlab environment is presented in Fig. 5

The evolution of the learning process can be traced by observing the changing values of errors made by the network in particular epochs of the learning process, calculated and averaged for the learning, validating and test sets, respectively (Fig. 6). It is worth noting that an 'epoch' in the process of learning means a time for showing the network all elements from the learning set. The learning process should be stopped when a error calculated for the validation set starts increasing with a decreasing error for the learning set, instead of decreasing. This is an overtraining effect. In Fig. 6 we can see that this moment took place in the 32 nd epoch of learning.

While observing errors in the learning process of a neural network (Fig. 6), attention should be paid to the fact that the vertical axis giving the mean-squared error for the 

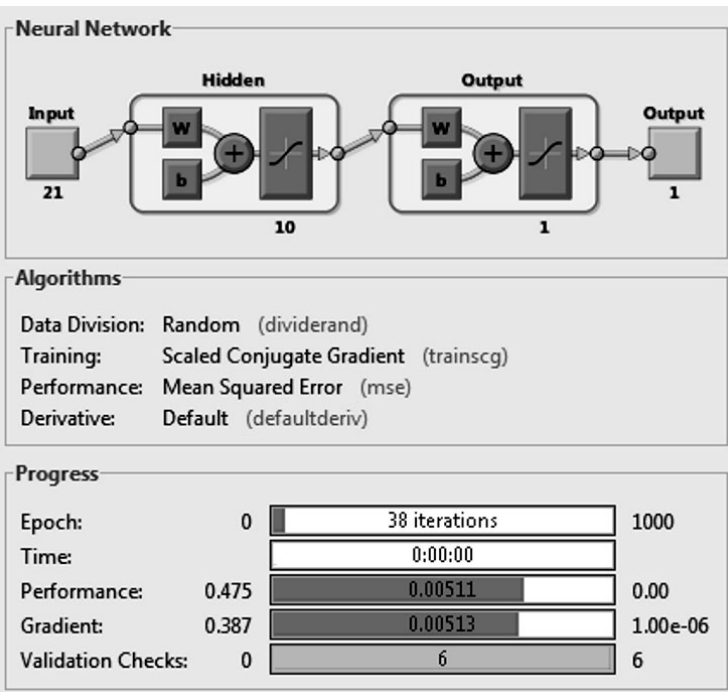

Fig. 5. Check-out panel of the Neural Network Toolbox while the network is learning.

respective three sets of data is scaled logarithmically. This gives an impression that the error decreases uniformly over the entire process of learning, while in reality a rapid drop in its value takes place at the initial stage of learning (first epochs) to significantly slow down later.

The plot in Fig. [ 6 shows changes (a decrease) in the mean error. For a full evaluation of the learning process, it is advantageous to show the errors made by the network for particular data (traces) in the process of learning. Such information is presented on the error histogram (Fig. 7), where the horizontal axis is scaled in values of the assumed measure of error, and the height of the blocks corresponds to the frequency of a given value of error (as shown on the horizontal axis). When drawing such a histogram, the data are divided into learning, validation and test ones.

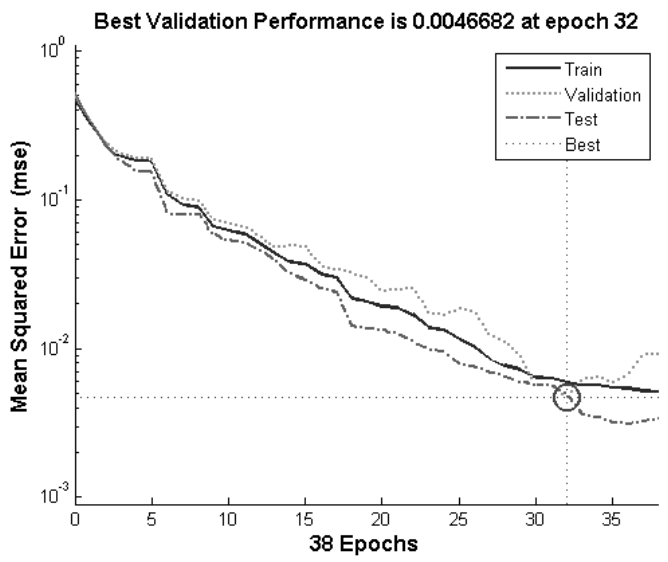

Fig. 6. Learning profiles.
The histogram presented in Fig. 7 shows that the predominant number of traces in the learning set as well as in the validation and test sets were recognized by the network with a very small error (the highest block is close to zero). Higher errors (both positive and negative) were rare and only in the learning set.

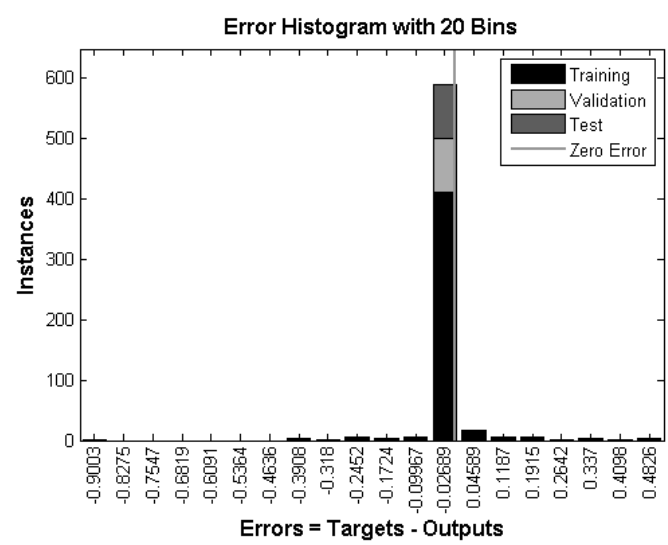

Fig. 7. Error histogram in the network at the final stage of learning.

\section{Evaluation of results of network performance and conclusions}

The task of the neural network analyzed in this paper was to ascribe information of an underground void or of a 'safe' (no void) geologic structure in a given area to each trace. The efficiency of realization of this task by a neural network can be best evaluated by showing (Fig.8) a 'confusion matrix', i.e., a table revealing how many times in recognitions provided by the network ('Output Class') an object belonging to class no. 1 (with void) actually corresponded to an area of the void in the analyzed trace (Target Class $=1$ ). Analogously, the correct recognitions corresponded to a situation of Output Class $=0$ when at the same time Target Class $=0$. The situations of Output Class $=0$, Target Class $=1$ and Output Class $=1$, Target Class $=0$ corresponded to errors. The confusion matrix tables are presented separately for the learning, validation and tests sets, and then also for all data jointly. Errors in the validation and test sets were absent, and in the learning set they did not exceed $0.5 \%$.

The high quality of data provided by the network can be also proved by its performance check-outs on sample traces, for which the polynomial coefficients are presented in Table 1. The probability of a void calculated for a trace no. 27 equalled 0.0465521619998640 , which was true as the trace actually stayed beyond the area in which the void was known to be present. The probability of a void for trace no. 330 was 0.943182754503623 , which corresponded to the void confirmed by a sinkhole. 

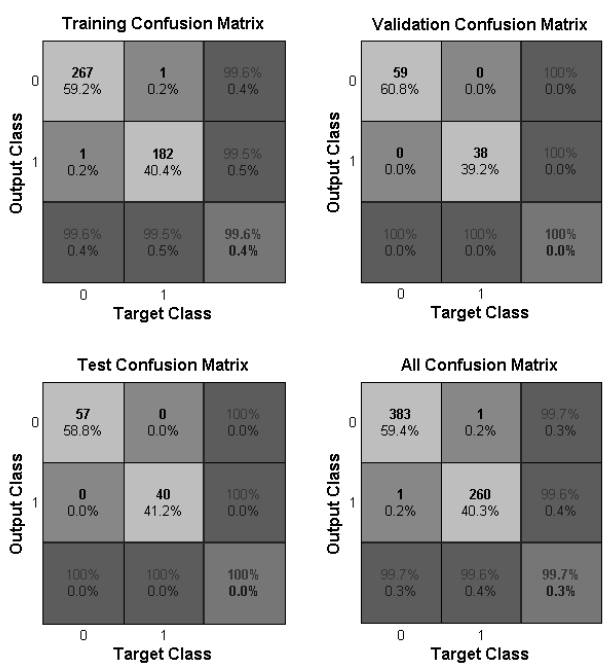

Fig. 8. Confusion matrix for the learning network.

Concluding, the neural network proved to be a useful tool aiding georadar images interpretation.

\section{Acknowledgment}

This work was financed with the funds of the National Science Center granted upon the decision no. DEC-2011/01/B/ST7/06178 under the contract no. UMO-2011/01/B/ST7/06178.

\section{References}

Marcak, H., Gołębiowski, T. and Tomecka-Suchoń, S. (2008). Geotechnical analysis and 4D GPR measurements for the assessment of the risk of sinkholes occurring in a Polish mining area, Near Surface Geophysics 6(4) 233-243.

McClymont, A.F., Green, A.G., Streich, R., Horstmeyer, H., Tronicke, J., Nobes, D.C., Pettinga, J., Campbell, J. and Langridge, R. (2008). Visualization of active faults using geometric attributes of 3D GPR data: An example from the alpine fault zone, New Zealand Geophysics 73(2): B11-B23.

Miaskowski, A. and Cieszczyk, S. (2011). Two-step inverse problem algorithm for ground penetrating radar technique, Przeglad Elektrotechniczny 87(12b): 22-24.
Tadeusiewicz, R. (2010). New trends in neurocybernetics, Computer Methods in Materials Science 10(1): 1-7.

Tadeusiewicz, R. (2011). Introduction to intelligent systems, in B.M. Wilamowski and J.D. Irvis (Eds.), Fault Diagnosis. Models, Artificial Intelligence, Applications, CRC Press, Boca Raton, FL, Chapter 1, pp. 1-1-1-12.

Tadeusiewicz, R., Chaki, R. and Chaki, N. (2014). Exploring Neural Networks with C\#, CRC Press, Boca Raton, FL.

Wei-Li, Huilin-Zhou and Xiaoting-Wan (2012). Generalized Hough transform and ANN for subsurface cylindrical object location and parameters inversion from GPR data, 14th International Conference on Ground Penetrating Radar GPR, Shanghai, China, pp. 281-285.

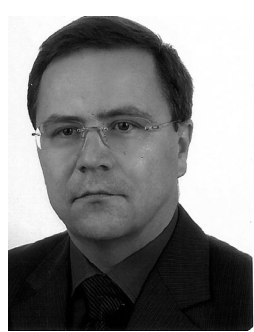

Piotr Szymczyk earned his M.Sc. degree in electronic engineering in 1988, and the Ph.D. degree in computer science in 1997 at the AGH University of Science and Technology (Kraków, Poland). Currently, he is a lecturer at AGH. His research interests include real time computer systems, embedded systems, natural computing and bioinformatics.

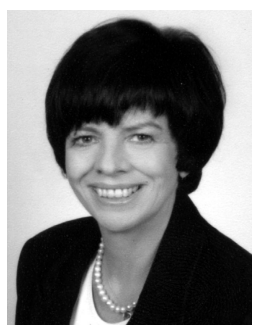

Sylwia Tomecka-Suchoń earned her Ph.D. and D.Sc. (habilitation) degrees at the AGH University of Science and Technology (Kraków, Poland). Currently, she is a lecturer at AGH. Her research interests include application of ground penetrating radars for monitoring near-surface strata properties changes resulting from mining, urban development and industrial processes.

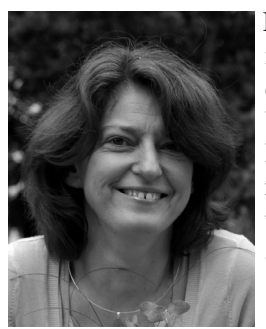

Magdalena Szymczyk earned her M.Sc. degree in electronic engineering in 1988 and the Ph.D. degree in computer science in 1999 at the AGH University of Science and Technology (Kraków, Poland). Currently, she is a lecturer at AGH. Her research interests include real time computer systems, embedded systems, parallel programming and bioinformatics.

Received: 7 July 2014 Revised: 14 October 2014 